\title{
Challenges related to testing of composite materials at high strain rates using the split Hopkinson bar technique
}

\author{
Ahmed Elmahdy*, and Patricia Verleysen \\ MST-DyMaLab research group, EEMMeCS Department, Faculty of Engineering and Architecture, Ghent University, \\ Tech Lane Ghent Science Park - Campus A, 9052 Zwijnaarde, Belgium
}

\begin{abstract}
The design of sample geometries and the measurement of small strains are considered the main challenges when testing composite materials at high strain rates using the split Hopkinson bar technique. The aim of this paper is to assess two types of tensile sample geometries, namely dog-bone and straight strip, in order to study the tensile behaviour of basalt fibre reinforced composites at high strain rates using the split Hopkinson bar technique. 2D Digital image correlation technique was used to study the distribution of the strain fields within the gauge section at quasi-static and dynamic strain rates. Results showed that for the current experiments and the proposed clamping techniques, both sample geometries fulfilled the requirements of a valid split Hopkinson test, and achieved uniform strain fields within the gauge section. However, classical Hopkinson analysis tends to overestimate the actual strains in the gauge section for both geometries. It is, therefore, important to use a local deformation measurement when using these 2 geometries with the proposed clamping technique.
\end{abstract}

\section{Introduction}

Composite materials have been increasingly used in many applications which can be subjected to impact loads, such as automotive and aeronautical applications. Consequently, it is important to study the behavior of such materials at high strain rates. Split Hopkinson bar setups have been typically used for that purpose, where high strain rates up to $8000 \mathrm{~s}^{-1}$ can be achieved [1]. Specimen design has been a critical element in high strain rate testing of composite materials with the split Hopkinson bar tensile technique, since its introduction by Kawata et al. [2]. Unlike static testing, dynamic testing involves dynamic stress waves traveling back and forth inside the specimen. The design of the tensile composite sample, therefore, has to achieve the following main requirements: (a) the specimen has to be short enough to achieve the required quasi-static stress equilibrium and to increase the achievable maximum strain rate, (b) a state of uniform stress and strain has to be achieved in the gauge section, (c) the design of the specimen has to promote failure within the gauge section, (d) the geometry has to be representative of the material constituents.

Dog-bone shaped specimens usually fulfill the above mentioned requirements, where the samples are reduced in width to create a transition zone between the gauge section and the shoulder section. A variety of dogbone samples with different dimensions were typically used [3-5], however, some limitations were reported such as the inability to achieve stress equilibrium at the beginning of the loading [6], and the tendency of failure due to stress concentrations in the transition zone [7]. Dog-bone samples which are reduced in the thickness direction rather than the width were also used by several researchers [8, 9]. Despite its effectiveness, it has been argued that reducing the thickness will reduce the areas which are rich in resin, and therefore change the overall representativeness of the tested material [10]. A compromise between the gauge length, width, and transition zone radius is, therefore, necessary to design a good dog-bone geometry for high strain rate tensile testing. Straight strip samples were also used [11, 12], however, they introduce a challenge related to clamping the sample on the input and the output bars for load introduction.

Another critical element for accurate characterization of composite materials using the split Hopkinson tensile bar technique is the accurate measurement of strains developing in the sample. Due to the general brittle nature of the composite materials, very small strains need to be measured. Dog-bone samples are usually known to cause an overestimation of the actual strains in sheet metal samples when using the classical Hopkinson strain analysis [13, 14]. This is particularly due to the effect of the transition zones, which increase the overall effective gauge length. Strain gauges are typically attached to the surface of the sample, however, they usually fail before the sample reaches the failure limit [6]. An optical, non-contact measurement technique, therefore, can be used as an alternative for accurate measurements of strains up to failure. Moreover, optical full field measurement techniques such as digital image correlation (DIC) enable accurate 
evaluation of the uniformity of the strain fields within the whole gauge area.

The aim of this paper is to assess 2 types of sample geometries - a dog-bone sample with uniform cross section and a straight strip sample - to be used in studying the tensile behavior of basalt fiber reinforced composites at high strain rates. The criteria for assessment includes the achievement of dynamic stress equilibrium and the achievement of uniform distribution of strains within the gauge section. Experiments are carried out using the split Hopkinson tensile bar setup of Ghent University. Reference static experiments are also carried out to evaluate the use of these geometries at quasi-static strain rates. Localized full strain fields are measured using 2D DIC technique in order to check the uniformity of strain distribution along the gauge section of both geometries. The stress-strain response and strain rate response obtained by classical Hopkinson analysis and high speed 2D DIC for both geometries is discussed and compared. This paper focuses on the linear elastic behavior of the basalt fiber reinforced composites up to a strain level of $2 \%$ for both static and dynamic experiments.

\section{Materials and geometries}

The tested material was a woven basalt fiber reinforced epoxy composite. Materials were supplied in the form of sheets with thickness $1.6 \mathrm{~mm}$, made up of 10 cross ply laminates. The distance between each two consecutive fiber tows is $2 \mathrm{~mm}$. Dog-bone samples (referred to as RG12) were cut to dimensions using water jet cutting, while straight strip samples (referred to as SSG) were cut using a water cooled cut-off wheel. All samples were extracted from the sheet in the fill direction (perpendicular to the main fiber warp direction). Aluminum tabs were glued at each side of the shoulders of the dog-bone specimens using a structural grade epoxy glue, to account for any possible premature failure in the shoulders. The straight strip samples were glued inside slotted aluminum tabs using a structural grade epoxy glue. Figures 1 and 2 show the dimensions of both the dog-bone and the straight strip samples respectively. The gauge length of both geometries was $10 \mathrm{~mm}$. In order to avoid any discrepancies related to sample geometries or clamping technique, the same geometries and clamping technique were used for both reference quasi-static and dynamic tensile experiments.

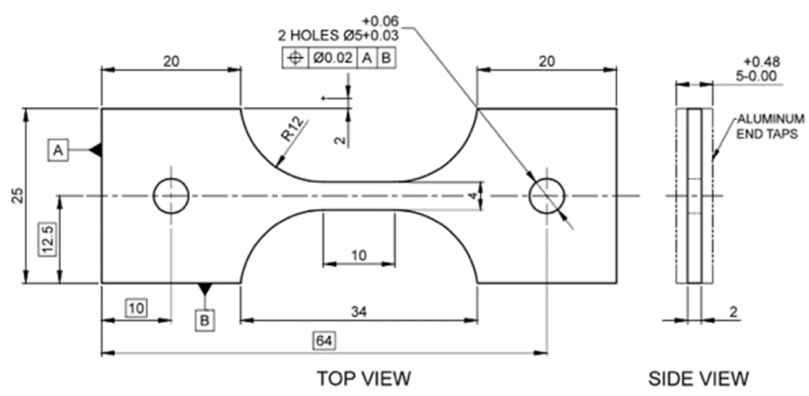

Fig. 1. Dog-bone (RG12) sample dimensions.
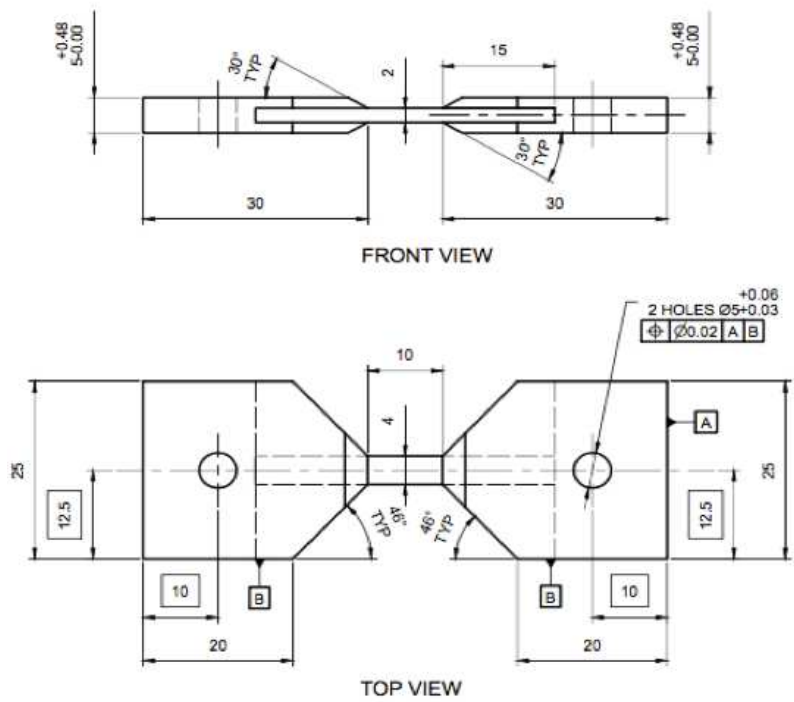

Fig. 2. Straight strip (SSG) sample dimensions.

\section{Experimental setups}

\subsection{Quasi-static setup}

Reference static tests were carried out using an Instron 5569 universal testing machine at a testing speed of 2 $\mathrm{mm} / \mathrm{min}$, corresponding to a strain rate of $0.0033 \mathrm{~s}^{-1}$. The load was measured using a $50 \mathrm{kN}$ load cell. Samples were placed between 2 long slotted bars using two $5 \mathrm{~mm}$ diameter dowel pins. 2D digital image correlation technique was used to measure localized strain fields on the surface of the samples. To this purpose, a 5 megapixel camera was used to capture images of the speckled sample during the deformation. The images were later processed to obtain full strain field measurements. Figure 3 shows the full reference static setup.

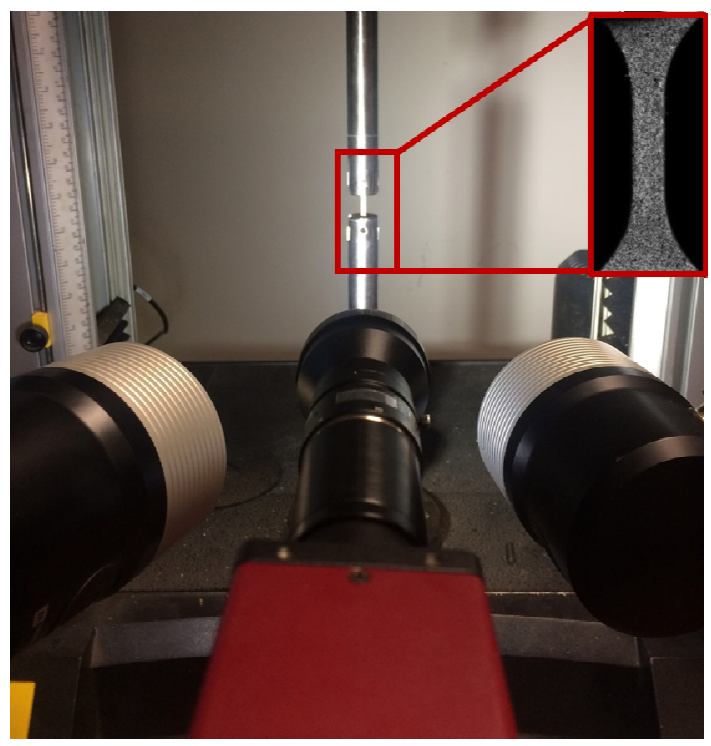

Fig. 3. Quasi-static setup. 


\subsection{Dynamic setup}

Dynamic experiments were carried out using a split Hopkinson tensile bar facility available at DyMaLab of Ghent University. The input and output bars were made of high strength aluminum with diameters of $25 \mathrm{~mm}$ and lengths of $6 \mathrm{~m}$ and $3 \mathrm{~m}$ respectively. The dynamic tensile stress wave was generated by accelerating an impactor towards a flange at the end of the input bar, at a speed of $8 \mathrm{~m} / \mathrm{s}$. Samples were placed between two slotted aluminum tabs and fixed using a $5 \mathrm{~mm}$ dowel pins. A special alignment device was used to ensure good alignment along the centerline of the specimen, the tabs, and the bars. In order to increase the accuracy of load measurements from the transmitted wave, next to traditional resistor gauges, two $120 \mathrm{ohm} \mathrm{KYOWA}$ semiconductor strain gauges (KSN-2-120-E4-11) were attached on the output bar. The semiconductor strain gauges could achieve a peak-to-peak strain resolution of 0.7 microstrains compared to a peak-to-peak strain resolution of 23 microstrains for foil type strain gauges. When dynamic stress equilibrium is achieved at the input and output bar interfaces, the average stress $(\sigma)$, strain $(\varepsilon)$ and strain rate $(\dot{\varepsilon})$ in the sample gauge section can be calculated from the classical Hopkinson bar equations using the reflected $\left(\varepsilon_{r}\right)$ and transmitted $\left(\varepsilon_{t}\right)$ strain measurements on the bars as follows:

$$
\begin{gathered}
\dot{\varepsilon}=2\left(\mathrm{C}_{0} / \mathrm{L}_{\mathrm{s}}\right) \cdot \varepsilon_{r}(t) \\
\varepsilon=2\left(\mathrm{C}_{0} / \mathrm{L}_{\mathrm{s}}\right) \cdot \int_{o}^{t} \varepsilon_{r}(t) \mathrm{d} t \\
\sigma=\mathrm{E}_{\mathrm{b}} \cdot\left(\mathrm{A}_{\mathrm{b}} / \mathrm{A}_{\mathrm{s}}\right) \cdot \varepsilon_{t}
\end{gathered}
$$

where $A_{b}$ and $A_{s}$ are the cross section area of the bars and the sample in $\mathrm{mm}^{2}$ respectively, $\mathrm{C}_{\mathrm{o}}$ is the elastic wave speed in the bar material, $\mathrm{L}_{\mathrm{s}}$ is the gauge length of the sample. A Photron AX200 Mini high speed camera was used to capture images of the speckled sample during deformation, at a speed of 86,400 frames per second, and at a resolution of $128 \times 288$ pixels. The images were later processed to obtain the full strain field measurements. Figure 4 shows the full dynamic tensile setup.

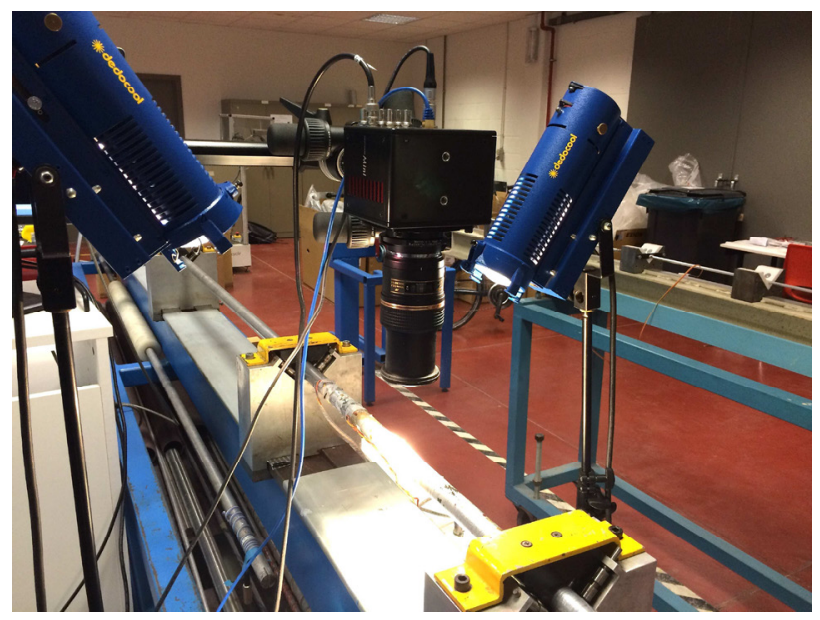

Fig. 4. Dynamic tensile setup.

\subsection{Data reduction for DIC}

All captured images were analyzed and processed using MatchID commercial digital image correlation software, in order to obtain the full strain fields during the static and dynamic experiments. Table 1 indicates the different processing parameters and correlation criterion used. Localized strains in the direction of loading $\left(\varepsilon_{D I C}\right)$ were calculated as average values taken at a gauge area of $8 \times 4$ $\mathrm{mm}$. The strain resolution for both low and high speed DIC systems was determined by calculating the average axial strain using 25 images taken at zero loading prior to starting the tests. The chosen processing parameters allowed to achieve a strain resolution of approximately 100 microstrains for both quasi-static and dynamic experiments. For the dynamic experiments, the strain rate based on DIC $\left(\dot{\varepsilon}_{D I C}\right)$ was calculated using finite difference method, derived from the following equation:

$$
\dot{\varepsilon}_{D I C}=\mathrm{d} \varepsilon_{D I C} / \mathrm{d} t
$$

Table 1. Processing Parameters for DIC.

\begin{tabular}{cc}
\hline Parameter & Value \\
\hline $\begin{array}{c}\text { Correlation } \\
\text { Criterion } \\
\text { Interpolation } \\
\text { Order } \\
\text { Shape function }\end{array}$ & $\begin{array}{c}\text { Zero normalized sum of square } \\
\text { differences (ZNSSD) }\end{array}$ \\
Subset size & Bicubic spline \\
Step size & Affine \\
Strain window size & $21 \times 21$ pixels \\
Strain convention & 10 Pixels \\
\end{tabular}

\section{Results and Discussion}

\subsection{Quasi-static results}

Figure 5 shows the quasi-static stress-strain response for the dog-bone RG12 sample based on 2D DIC strain measurements for a gauge area of $8 \times 4 \mathrm{~mm}$. In order to study the uniformity of the strain fields within the gauge section, average strain fields were also extracted for a gauge area of $5 \times 4 \mathrm{~mm}$ and compared to that of area $8 \times 4$ $\mathrm{mm}$. It can be clearly seen that nearly identical strains were obtained for both extractions. The indicated strain fields up to $2 \%$ strain also show the strain uniformity within the gauge section.

To further study the contribution of the transition zone to the total strain of a statically deformed sample up to a test duration of 4 seconds (corresponding to $1.5 \%$ strain), axial strains were extracted along a vertical central line, which passes through the transition zones and the gauge section, as shown in figure 6 . The 
contribution of the transition zone is nearly equal to that of the gauge section during the progress of the deformation. This is mainly related to the linear elastic behaviour of the basalt epoxy composite up to $2 \%$ strain, as opposed to metals where plasticity plays a role in concentrating the deformation within the gauge section. The present irregularities in the strain data is due the weaving (interlocking) nature of the fabric tows.

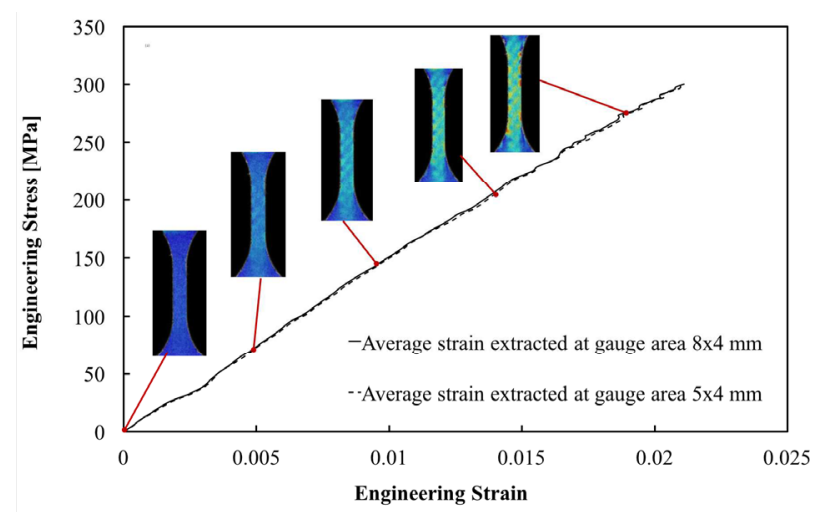

Fig. 5. Strain uniformity in gauge section for RG12 geometry at quasi-static strain rates as proven by DIC processing.

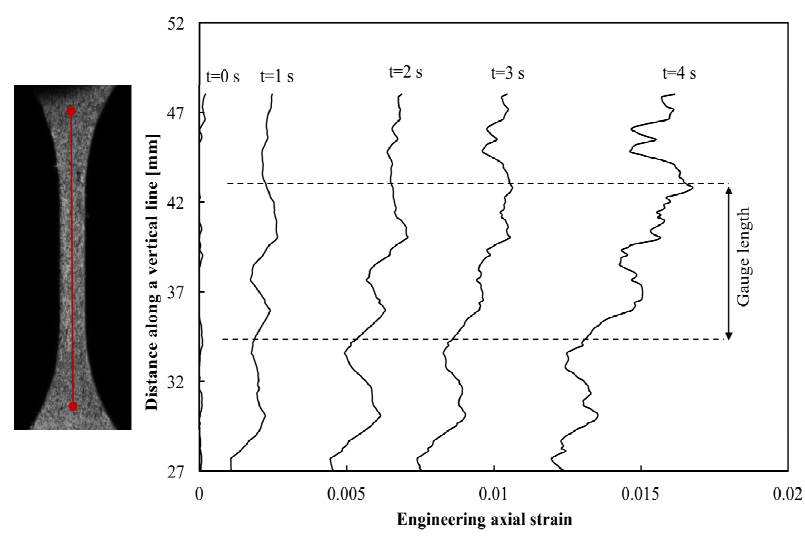

Fig. 6. Axial strain distribution alone a vertical centreline for RG12 geometry at quasi-static strain rates.

Figure 7 shows the quasi-static stress - strain response for the straight strip SSG sample geometry based on 2D DIC measurements. Similar to the RG12 geometry, average axial strains were extracted at gauge areas of $8 \times 4 \mathrm{~mm}$ and $5 \times 4 \mathrm{~mm}$ to study the strain uniformity along the gauge section. It can be seen that both extractions show good agreement. The strain fields at different stress levels also show a state of uniform strain within the gauge section. Strain concentrations begin to develop with the progression of deformation due to stress concentration effects at the ends of the aluminium tabs.

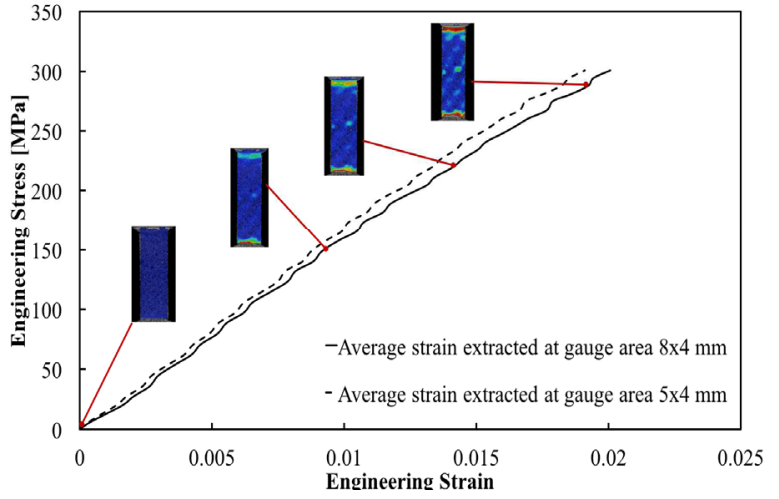

Fig. 7. Strain uniformity for SSG12 geometry at quasi-static strain rates.

Figure 8 shows a comparison between the stressstrain response obtained by both dog-bone RG12 and straight strip SSG samples geometries at quasi-static strain rates, based on 2D DIC strain measurements. It can be seen that the response for both geometries shows very good agreement, despite the slight tendency of overestimation of strains for the dog-bone sample geometry compared to the straight strip geometry.

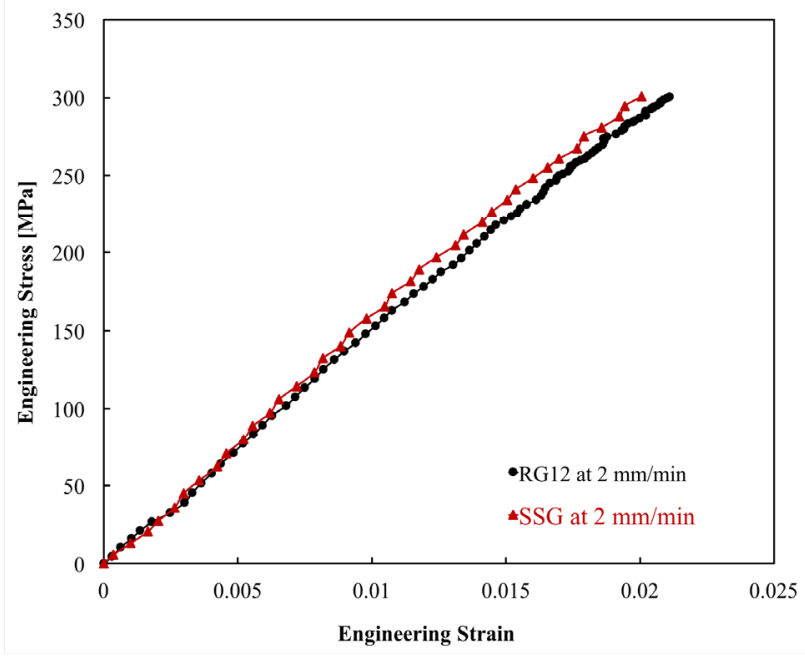

Fig. 8. Quasi-static stress - strain response of basalt epoxy composite for both RG12 and SSG sample geometries.

\subsection{Dynamic results}

Dynamic stress equilibrium was checked for all dynamic experiments by calculating and comparing the forces at the interfaces of input and output bars. Both dog-bone RG12 geometry and straight strip SSG geometry achieved the required dynamic stress equilibrium throughout the experiment.

Figure 9 represents the high strain rate stress-strain response of basalt epoxy composites using the dog-bone RG12 geometry, based on high speed 2D DIC strain measurements. Average axial strains were extracted at gauge areas $8 \times 4$ and $5 \times 4 \mathrm{~mm}$ respectively to check the uniformity of the strains within the gauge section. Both strain extractions show very good agreement, indicating 
that the generated strain fields are indeed homogeneous along the gauge length.

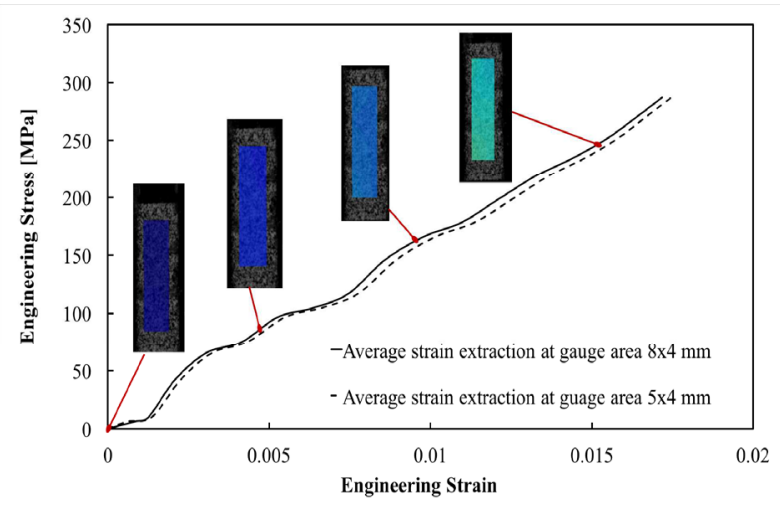

Fig. 9. Strain uniformity for RG12 geometry at dynamic strain rates.

The same strain uniformity along the gauge section can also be seen for the straight strip SSG geometry, as shown in figure 10 .

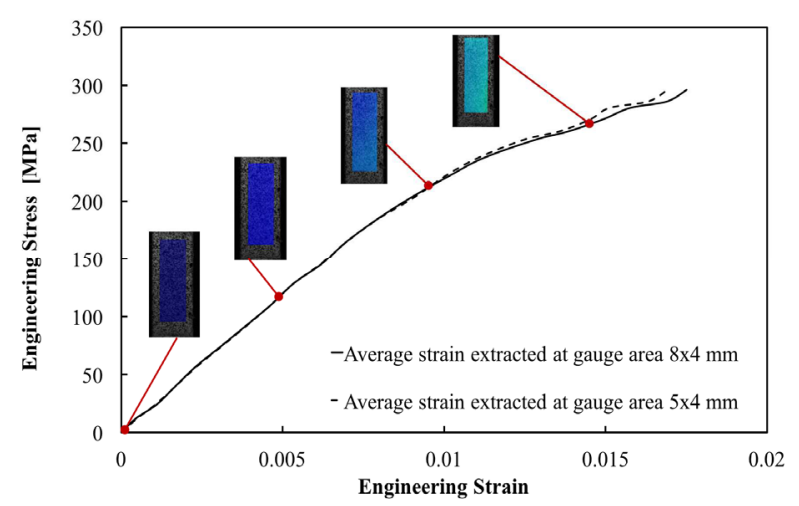

Fig. 10. Strain uniformity for SSG geometry at dynamic strain rates.

Figure 11 shows the high strain rate tensile behaviour of basalt epoxy composite for the dog-bone RG12 geometry using classical Hopkinson analysis and the high speed DIC measurements. The strains obtained by classical Hopkinson analysis are overestimated by a factor of approx. 3 compared to the strains obtained by high speed 2D DIC technique. This is due to the use of the classical Hopkinson analysis with respect to the current pin clamping technique. In the classical Hopkinson analysis, the measured strain waves are shifted to the bar interfaces at the centre of the dowel pins, where the actual transfer of the load to the sample takes place. This region between the centres of the dowel pins represents an apparent gauge length, where the calculated total strain includes not only the strains within the gauge section, but also strains outside the gauge section in the pins, aluminium bars, and the transition zones. The use of a smaller actual gauge length in the calculation of strains results in the observed overestimation in the strains. The same overestimation factor can also be seen for the straight strip SSG geometry in figure 12. In the case of the SSG geometry, deformations of the pins and the aluminium tabs are also included within the apparent gauge length, resulting in the overestimation of strains.

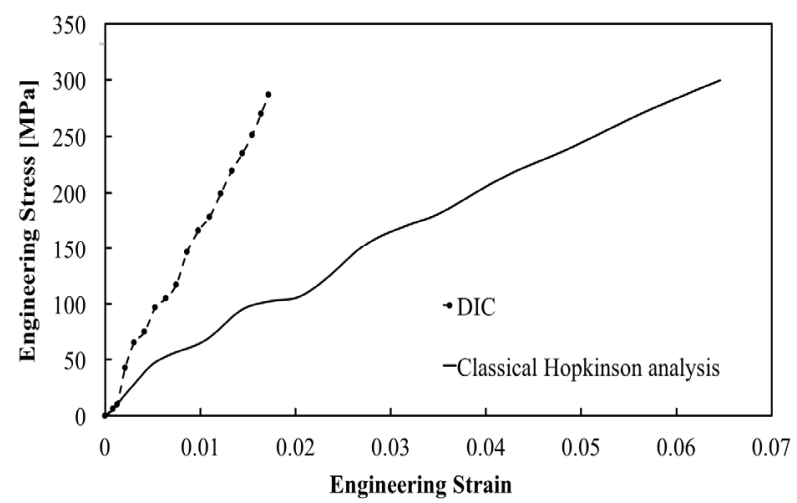

Fig. 11. Stress-strain response of basalt epoxy using RG12 geometry based on 2D DIC and classical Hopkinson analysis.

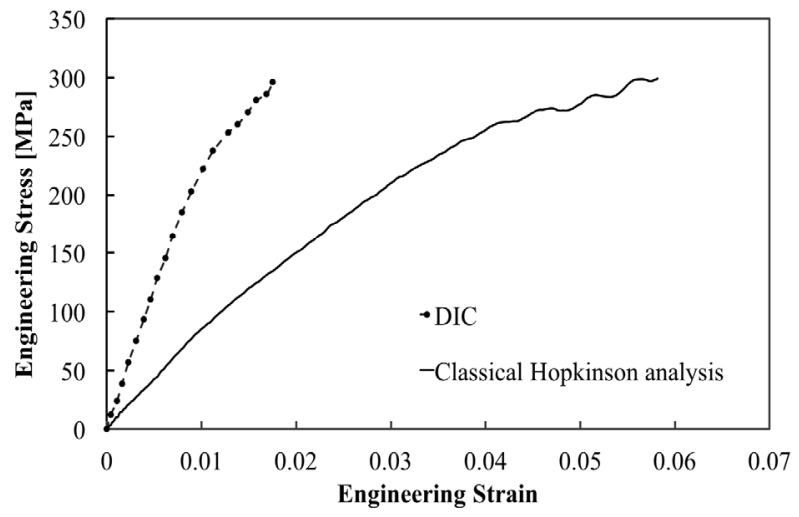

Fig. 12. Stress-strain response of basalt epoxy using SSG geometry based on 2D DIC and classical Hopkinson analysis.

Figure 13 shows the average strain rates achieved using the high speed 2D DIC technique and the classical Hopkinson analysis for both geometries. Using classical Hopkinson analysis, the average strain rate achieved was $300 \mathrm{~s}^{-1}$ for the RG12 geometry and $310 \mathrm{~s}^{-1}$ for the SSG geometry. However, the average strain rates achieved using the local, high speed 2D DIC technique were $80 \mathrm{~s}^{-1}$ for the RG12 geometry, and $90 \mathrm{~s}^{-1}$ for the SSG geometry. The average strain rates obtained by the classical Hopkinson analysis is overestimated by a factor of approx. 3 compared to the strain rates achieved using the high speed 2D DIC technique for both geometries. This is also due to the use classical Hopkinson analysis with the pin clamping technique. 


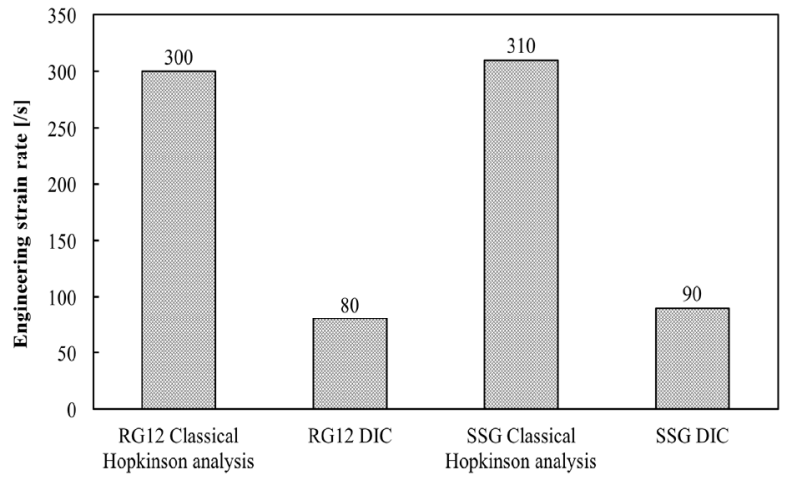

Fig. 13. Achieved strain rates of basalt epoxy using SSG and RG12 geometries based on 2D DIC and classical Hopkinson analysis.

Figure 14 shows a comparison between the stressstrain response of both RG12 and SSG tensile geometries at high strain rates. Considering the dynamic nature of the tests, the two geometries show good agreement in dynamic stress-strain response.

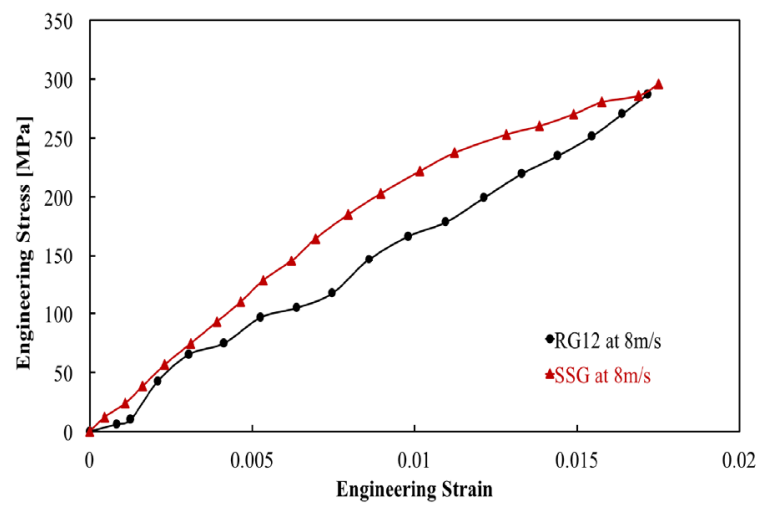

Fig. 14. Quasi-static stress - strain response of basalt epoxy composite for both RG12 and SSG sample geometries.

\section{Conclusion}

In order to study the tensile behaviour of basalt fiber reinforced composites at low and high strain rates, 2 samples geometries were compared and assessed in terms of dynamic stress equilibrium and the distribution of strains along the gauge section. 2D DIC technique was used to measure the full strain fields developed along the gauge section. Considering the testing conditions and clamping techniques presented in this study, the following can be concluded:

1- Both sample geometries achieve the main requirements of a valid split Hopkinson tensile bar test, which are the dynamic stress equilibrium and uniform strain fields along the gauge section up to $2 \%$ strain.

2- At quasi-static strain rates, the total, axial deformation of the transition zones of the dogbone RG12 geometry was nearly equal to the deformation of the gauge section, which causes a significant overestimation of the material strains if the total sample deformation is used to calculate the strain in the gauge section.

3- Classical Hopkinson analysis also overestimates the actual strains and strain rates in the sample by approx. a factor of 3 for both geometries. Localized measurement of the strains along the gauge section is, therefore, crucial for accurate measurement of the high strain rate behaviour of composites.

The project "EXTEME" leading to this paper has received funding from the European Union's Horizon 2020 research and innovation program under agreement No. 636549.

\section{References}

1. W. Sharpe, ed. Springer handbook of experimental solid mechanics. Springer Science \& Business Media (2008)

2. K. Kawata, A. Hondo, S. Hashimoto, N. Takeda, and H. L. Chung. Proc of Jap-US Conf on Comp Mat, Jap. Soc. for Compos. Mater., Tokyo (1981)

3. R. Foroutan, J. Nemes, H. Ghiasi, P. Hubert. Compos. Struct., 106 (2013)

4. J. Rodriguez, I. Chocron, M. Martinez, and V. Sanchez-Galvez, Composites Part B 27, 2 (1996)

5. P. Ma, H. Hu, L. Zhu, B. Sun, and B. Gu., J. Compos. Mater. 45, 24 (2011)

6. T. Gomez-del Rio, E. Barbero, R. Zaera, and C. Navarro, Compos. Sci. Technol. 65, 1 (2005)

7. G. Reyes, U. Sharma. Proc of SPE Auto. Comp. Conf. and Exhib., Detroit, MI. (2009)

8. J. Harding, L. Welsh. J. Mater. Sci. 18, 6 (1983)

9. L. Welsh, J. Harding. Le Journal de Physique Colloques 46, no. C5 (1985)

10. J. Hodgkinson, ed. Mechanical testing of advanced fibre composites. Elsevier (2000)

11. N. Taniguchi, T. Nishiwaki, H. Kawada. Adv. Compos. Mater.16, 2 (2007)

12. K. Suga, K. Okamoto, K. Takagi, H. Nakatani, S. Ogihara, M. Kikuchi. Adv. Compos. Mater. 21, 3 (2012)

13. P. Verleysen, J. Degrieck, T. Verstraete, J. Van Slycken. Exp. Mech. 48, 5 (2008)

14. A. Gilat, T. Schmidt, A. Walker. Exp. Mech. 49, 2 (2009) 